\title{
Entangled State Creation by a Nonlinear Coupler Pumped in Two Modes
}

\author{
V. Le Duc ${ }^{1}$, V. Cao Long ${ }^{2 *}$ \\ ${ }^{1}$ Faculty of Natural Sciences, Hong Duc University \\ Quang Trung 665, 450000 Thanh Hoa, Vietnam \\ ${ }^{2}$ Quantum Optics and Engineering Division, Institute of Physics, University of Zielona Góra \\ A. Szafrana 4a, 65-516 Zielona Góra, Poland \\ *E-mail: V.CaoLong@if.uz.zgora.pl
}

Received: 07 December 2016; revised: 19 December 2016; accepted: 20 December 2016; published online: 31 December 2016

\begin{abstract}
In this paper we consider a system with two nonlinear oscillators which are coupled via a nonlinear interaction. In order to excite the system, we use two external coherent fields. Two oscillators have different frequencies. It follows from numerical simulation that evolution of the system is similar to that of a combination of n-photon states. Therefore, the considered system behaves as so-called nonlinear quantum scissor. Nevertheless, evolution of the system generates Bell-like states in several times with very high probability. Because of the nonlinear properties of oscillators and their interaction, the system creates a truncation of optical states, which leads to obtain two-qubit states. It will also be shown that these states appear several times in the qutrit-qutrit system.
\end{abstract}

Key words: entanglement, quantum scissor, Bell-like states, Kerr-like systems

\section{INTRODUCTION}

Quantum information and quantum computation belong to the field that concentrates studies of physicists for several decades. There are numerous published papers in this area and some of them are in the form of monographs $[1,2]$. One of the central problems in this field is finding mechanisms to generate entanglement states in proper physical systems. The first task is find how to make a system which can create a set of n-photon states. For this purpose several interesting nonlinear optical phenomena are used, in particular the Kerr effect that is created by proper physical systems, named usually as nonlinear Kerr-like systems. These systems are well-known in literature. They consist of two nonlinear components that interact with each other in a nonlinear way. Using laser to impact optical systems is a popular method to seek special phenomena in nonlinear optics. Thus one can obtain the systems which are able to create a finite number of states from the states in the infinite-dimension Hilbert space. Such systems are known as quantum scissors. The idea of quantum scissors has been initiated by Jensen and Maier, and later developed by other authors to construct the linear quantum scissors [3,4] or nonlinear quantum scissors [5-7], depending on the used optical elements and the type of their interaction. Recently, some special systems are considered, in which the interaction between two nonlinear oscillators can be modeled with use of Dirac - delta function [8,9] or Werner-like states [10]. The most important result of these considerations is the generation of Bell-like states - the maximally entangled states. In these papers some interesting phenomena such as sudden death and birth of entanglement [11] or an exciting field phase effect [12] have been discovered. In this paper we propose a system which can also achieve the Bell-like states, but it has several special characteristics that are different from others. Our system is a nonlinear coupler pumped in two modes with nonlinear interaction between oscillators involved in 
the system. We show that under appropriate conditions the time evolution of the system is restricted to a superposition of vacuum, single-photon and two-photons states, so the proposed system acts as a nonlinear quantum scissor. Next, we compare our results with that obtained for systems in which interaction between oscillators is linear pumped in two modes [5,6], and also interaction between oscillators is nonlinear pumped in one modes [7]. We show that our system creates the state that has better entanglement than that obtained in [7] with the same values of parameters involved in the problem. Moreover, we realize that the result obtained by our system does not depend on phase deviation between two external fields that are pumped to modes. This result is more valuable in comparison with the case considered in [5].

\section{THE MODEL}

Our model is based on two oscillators which are characterized by Kerr nonlinearities $\chi_{a}$ and $\chi_{b}$ with the field modes $a$ and $b$, respectively. The oscillators are located in a high-Q cavity and they interact with themselves in a nonlinear way. Moreover, in the model, two field modes corresponding to two Kerr - like oscillators $\mathrm{a}$ and $\mathrm{b}$ are pumped by two external classical fields, so the model is different from that in [8]. In the interaction picture, the effective Hamiltonian describing the system has the following form

$$
\hat{H}=\hat{H}_{N L}+\hat{H}_{\text {inter }}+\hat{H}_{\text {extra }},
$$

where

$$
\begin{gathered}
\hat{H}_{N L}=\hat{H}_{N L(a)}+\hat{H}_{N L(b)}=\frac{\chi_{a}}{2}\left(\hat{a}^{\dagger}\right)^{2} \hat{a}^{2}+\frac{\chi_{b}}{2}\left(\hat{b}^{\dagger}\right)^{2} \hat{b}^{2}, \\
\hat{H}_{\text {inter }}=\epsilon\left(\hat{a}^{\dagger}\right)^{2} \hat{b}^{2}+\epsilon^{*}\left(\hat{b}^{\dagger}\right)^{2} \hat{a}^{2}, \\
\hat{H}_{\text {extra }}=\alpha \hat{a}^{\dagger}+\alpha^{*} \hat{a}+\beta \hat{b}^{\dagger}+\beta^{*} \hat{b} .
\end{gathered}
$$

In expression (1), the component $\hat{H}_{N L}$ describes nonlinear oscillators in two modes $a$ and $b, \hat{H}_{\text {inter }}$ is a term of Hamiltonian that describes interaction between the modes, whereas the component $\hat{H}_{\text {extra }}$ corresponds to interaction of the modes with external classical fields. $\hat{a}^{\dagger}$ and $\hat{b}^{\dagger}$ are boson creation operators corresponding to two mode $a$ and $b$ while $\hat{a}$ and $\hat{b}$ are boson annihilation operators, respectively. The parameter $\epsilon$ is a constant which represents the strength of internal interaction between two oscillators in the model. In order to describe strength of coupling between the modes $a$ and $b$ with external classical fields, we use complex parameters $\alpha$ and $\beta$, respectively. It is worth noting that the component $\hat{H}_{\text {inter }}$ does not have the terms containing $\hat{a}^{\dagger} \hat{a} \hat{b}^{\dagger} \hat{b}$ elements and their combination.

In this section, we restrict ourselves to the case without damping. Then evolution of the system is described by time-dependent wave function which is determined by the Schrödinger equation in interaction picture

$$
i \frac{d}{d t}|\psi(t)\rangle=\hat{H}|\psi(t)\rangle
$$

where, wave function $|\psi(t)\rangle$ describing evolution of the system can be expanded in the n-photon Fock states with complex probability amplitudes $c_{m n}(t)$ in the following form

$$
|\psi(t)\rangle=\sum_{m, n=0}^{\infty} c_{m n}(t)|m\rangle_{a}|n\rangle_{b} .
$$

Substituting (6) into (5) we get set of equations of motion for $c_{m n}(t)$ (use units $\hbar=1$ )

$$
\begin{aligned}
i \frac{d}{d t} c_{m n}(t) & =\left[\frac{1}{2} \chi_{a} m(m-1)+\frac{1}{2} \chi_{b} n(n-1)\right] c_{m n}(t) \\
& +\varepsilon \sqrt{(n+2)(n+1) m(m-1)} c_{m-2, n+2}(t) \\
& +\varepsilon^{*} \sqrt{(m+2)(m+1) n(n-1)} c_{m+2, n-2}(t) \\
& +\alpha^{*} \sqrt{m+1} c_{m+1, n}(t)+\alpha \sqrt{m} c_{m-1, n}(t) \\
& +\beta^{*} \sqrt{n+1} c_{m, n+1}(t)+\beta \sqrt{n} c_{m, n-1}(t) .
\end{aligned}
$$

Because our system consists of two oscillators which are pumped by external classical coherent fields, there is not conservation of the total energy of the system. Thus, the number of photon can increase in the modes. As a result, the system dynamics will include Fock states of an infinite number of photons. Nevertheless, if we suppose that the external coherent fields that pump into modes are weak and have a constant strength, we can use the approach given in the formalism of nonlinear quantum scissors. This approach is discussed widely in [6-8]. Accordingly to this, the wave function (6) can be truncated to the wave function that is described by only by some Fock states. It follows from the form of the nonlinear component of Hamiltonian $\hat{H}_{N L}$ that the wave function of system can be expanded only to four states: $|0\rangle_{a}|2\rangle_{b},|2\rangle_{a}|0\rangle_{b},|1\rangle_{a}|2\rangle_{b}$ and $|2\rangle_{a}|1\rangle_{b}$. Therefore, the wave function can be written in the form

$$
\begin{aligned}
|\psi(t)\rangle_{c u t} & =c_{02}(t)|0\rangle_{a}|2\rangle_{b}+c_{12}(t)|1\rangle_{a}|2\rangle_{b} \\
& +c_{21}(t)|2\rangle_{a}|1\rangle_{b}+c_{20}(t)|2\rangle_{a}|0\rangle_{b} .
\end{aligned}
$$




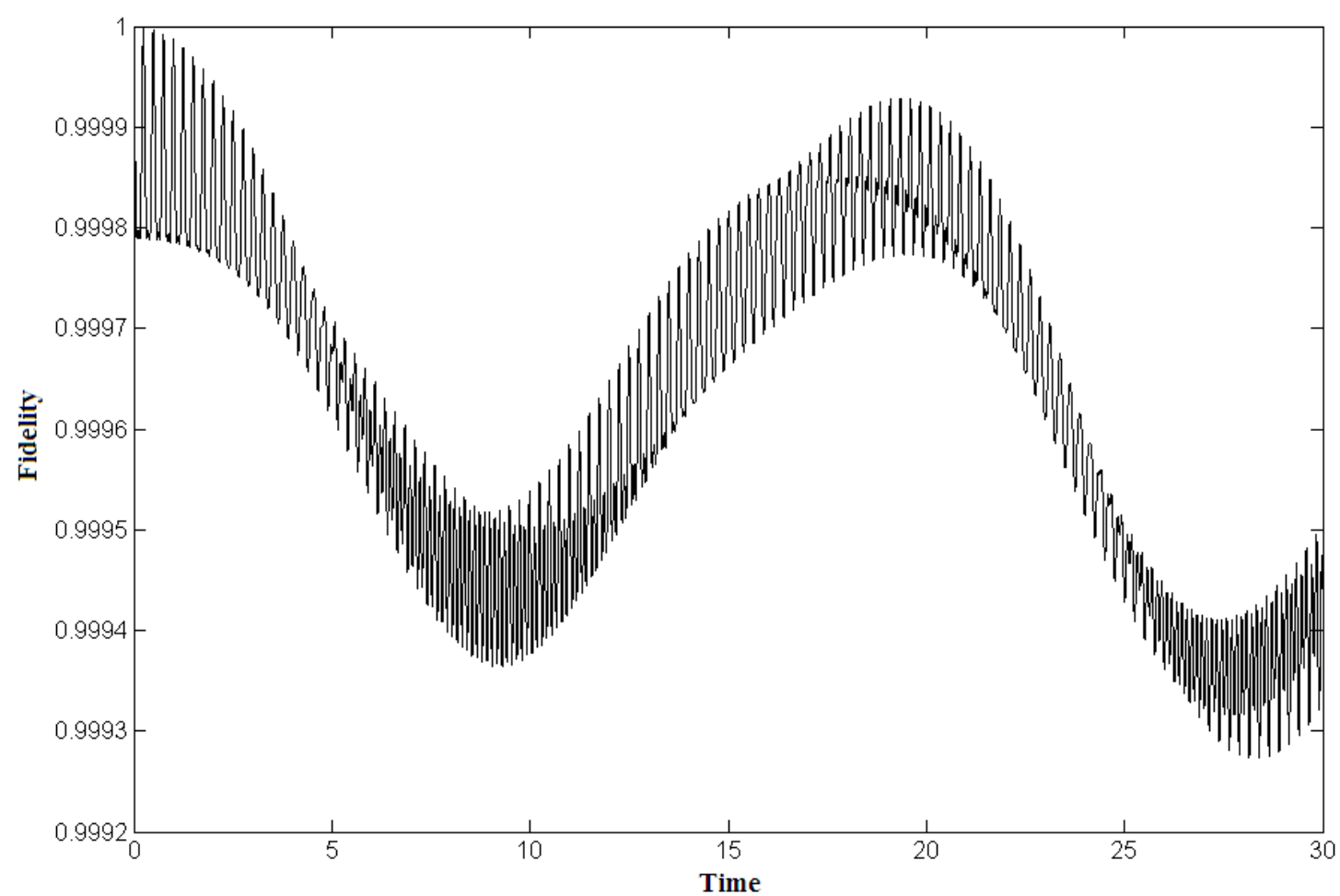

Fig. 1. The time-evolution of fidelity $\mathbf{F}$. The coupling strengths $\alpha=\frac{\pi}{25} \mathrm{rad} / \mathrm{s}, \epsilon=\frac{\pi}{25} \mathrm{rad} / \mathrm{s}$ and the nonlinearities $\chi_{a}=\chi_{b}=25 \mathrm{rad} / \mathrm{s}$. Time unit is scalled in $1 / \chi$

Using (7) we can obtain equations for complex probability amplitudes $c_{02}(t), c_{12}(t), c_{21}(t)$ and $c_{20}(t)$ as follows

$$
\begin{aligned}
i \frac{d}{d t} c_{02}(t) & =2 \varepsilon^{*} c_{20}(t)+\alpha^{*} c_{12}(t), \\
i \frac{d}{d t} c_{12}(t) & =\alpha c_{02}(t), \\
i \frac{d}{d t} c_{21}(t) & =\alpha c_{20}(t), \\
i \frac{d}{d t} c_{20}(t) & =2 \varepsilon c_{02}(t)+\alpha c_{21} .
\end{aligned}
$$

The equations (9) can be solved if we know the initial state of the system. We suppose that the initial state (in $\mathrm{t}=0$ ) of the system has two photons in mode $a$ and no photon in mode $b$ in the cavity. It means that we have $c_{02}(0)=c_{12}(0)=c_{21}(0)=0$ and $c_{20}(0)=1$. Moreover, we assume that two external coherent fields have the same strength, i.e., the parameters $\alpha$ and $\beta$ are real numbers and $\alpha=\beta$. Then, solution of equations (9) is obtained exactly in the following analytical form:

$$
c_{20}(t)=\frac{1}{2 \lambda}\left[\left(\lambda+\varepsilon^{2}\right) \cos \left(\mu_{1} t\right)+\left(\lambda-\varepsilon^{2}\right) \cos \left(\mu_{2} t\right)\right],
$$

$$
\begin{aligned}
c_{21}(t) & =\frac{-i}{2 \alpha \lambda}\left[\left(\lambda-\varepsilon^{2}\right) \mu_{1} \sin \left(\mu_{2} t\right)\right. \\
& \left.+\left(\lambda+\varepsilon^{2}\right) \mu_{2} \sin \left(\mu_{1} t\right)\right], \\
c_{12}(t) & =\frac{\alpha \varepsilon}{2 \lambda}\left[\cos \left(\mu_{1} t\right)-\cos \left(\mu_{2} t\right)\right], \\
c_{02}(t) & =\frac{-i \varepsilon}{2 \lambda}\left[\mu_{1} \sin \left(\mu_{1} t\right)-\mu_{2} \sin \left(\mu_{2} t\right)\right],
\end{aligned}
$$

where

$$
\begin{aligned}
& \lambda=\varepsilon \sqrt{\alpha^{2}+\varepsilon^{2}}, \\
& \mu_{1}=\sqrt{\alpha^{2}+2 \varepsilon^{2}+\lambda}, \\
& \mu_{2}=\sqrt{\alpha^{2}+2 \varepsilon^{2}-\lambda} .
\end{aligned}
$$

To explore these analytical results, we used a numerical method to calculate the fidelity of output state $\hat{\rho}(t)=$ $|\psi(t)\rangle\langle\psi(t)|$. Here, the wave function $|\psi(t)\rangle$ is determined directly from

$$
|\psi(t)\rangle=\exp (-i \hat{H} t)|2\rangle_{a}|0\rangle_{b} .
$$

Then, we calculate the closeness of two quantum states, namely between $|\psi(t)\rangle$ and truncated $|\psi(t)\rangle_{\text {cut }}$ for showing 

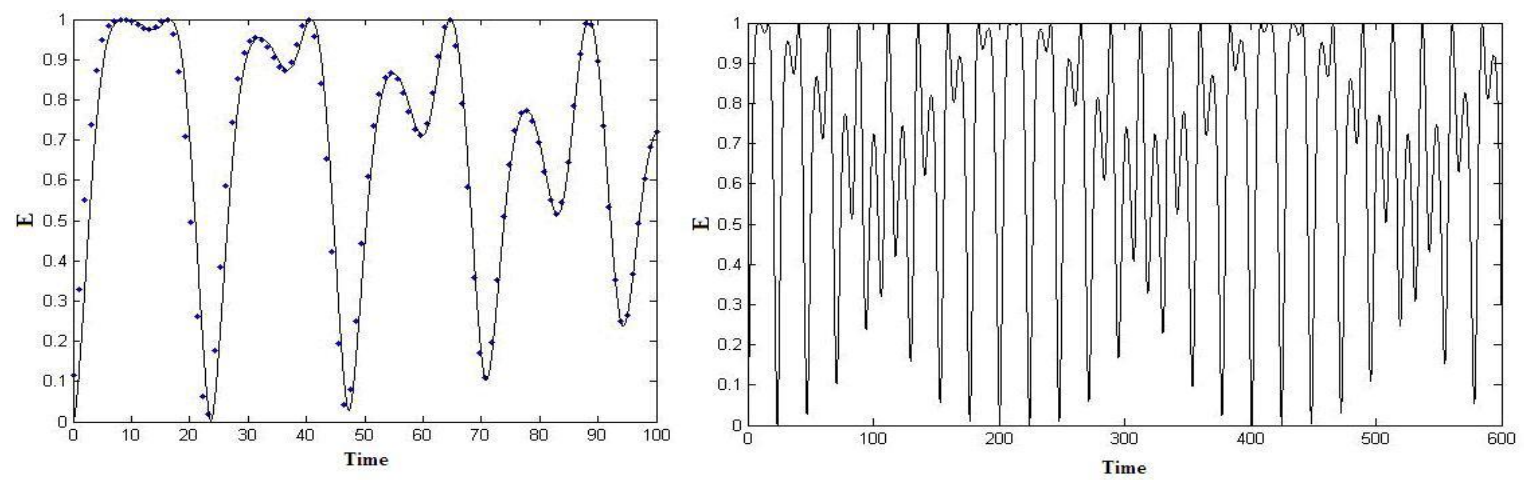

Fig. 2. Time-evolution of entropy of entanglement $\mathbf{E}$ of the generated $|\psi(t)\rangle$ (dots) and the truncated state $|\psi(t)\rangle_{\text {cut }}$ (solid curve). The coupling strengths $\alpha=\frac{\pi}{25} \mathrm{rad} / \mathrm{s}, \epsilon=\frac{\pi}{25} \mathrm{rad} / \mathrm{s}$ and the nonlinearities $\chi_{a}=\chi_{b}=25 \mathrm{rad} / \mathrm{s}$. Time unit is scalled in $1 / \chi$

that the truncation is ideally made. The quantity measuring this closeness is so-called fidelity $\mathrm{F}$ which has been defined clearly in [6] and [7]. The result of calculation is shown in Fig. 1.

In more detail, we can see from Fig. 1, which presents the time evolution of fidelity, that fidelity of $|\psi(t)\rangle_{\text {cut }}$ has values approximally to unity. The declination of fidelity from unity is only smaller than $10^{-3}$. It means that description of time evolution of the considered system by means of analytical expression (8) is quite good. In the further part hereof we will use these analytical results to analyze the generation of Bell-like states.

\section{EVOLUTION OF SYSTEM AND EXHIBITION OF BELL-LIKE STATES}

This section will be devoted to characterize the states which are created by our model. An important feature of this model is its ability to make maximally entangled states, the output state $|\psi(t)\rangle_{\text {cut }}$. To show the entanglement of the state $|\psi(t)\rangle_{\text {cut }}$, we describe time-evolution of entanglement in terms of the von Neumann entropy, that is defined in [1]. With the state $|\psi(t)\rangle_{\text {cut }}$ in (8), the full density matrix $\rho_{a b}=|\psi\rangle_{\text {cutcut }}\langle\psi|$ shows the time-evolution of the system in time. The partial trace of $\rho_{a b}$ with respect to the mode $b$ is

$$
\begin{aligned}
\rho_{b} & =\operatorname{Tr}_{a} \rho_{a b}=\left|c_{20}\right|^{2}|0\rangle_{b b}\left\langle 0\left|+c_{20} c_{21}^{*}\right| 0\right\rangle_{b b} \\
& \left\langle 1\left|+c_{21} c_{20}^{*}\right| 1\right\rangle_{b b}\left\langle\left. 0|+| c_{21}\right|^{2} \mid 1\right\rangle_{b b}\langle 1| \\
& +\left(\left|c_{02}\right|^{2}+\left|c_{12}\right|^{2}\right)|2\rangle_{b b}\langle 2| .
\end{aligned}
$$

Then, the entropy von Neumann (that can be seen as one of the measures of entanglement of the system) is

$$
\begin{aligned}
E & =-\operatorname{Tr} \rho_{a} \log _{2} \rho_{a}=-\operatorname{Tr} \rho_{b} \log _{2} \rho_{b}= \\
& -\lambda_{1} \log _{2} \lambda_{1}-\lambda_{2} \log _{2} \lambda_{2},
\end{aligned}
$$

where $\lambda_{1}$ and $\lambda_{2}$ are eigenvalues of $\rho_{b}$. The entropy of entanglement is presented in the Fig. 2.

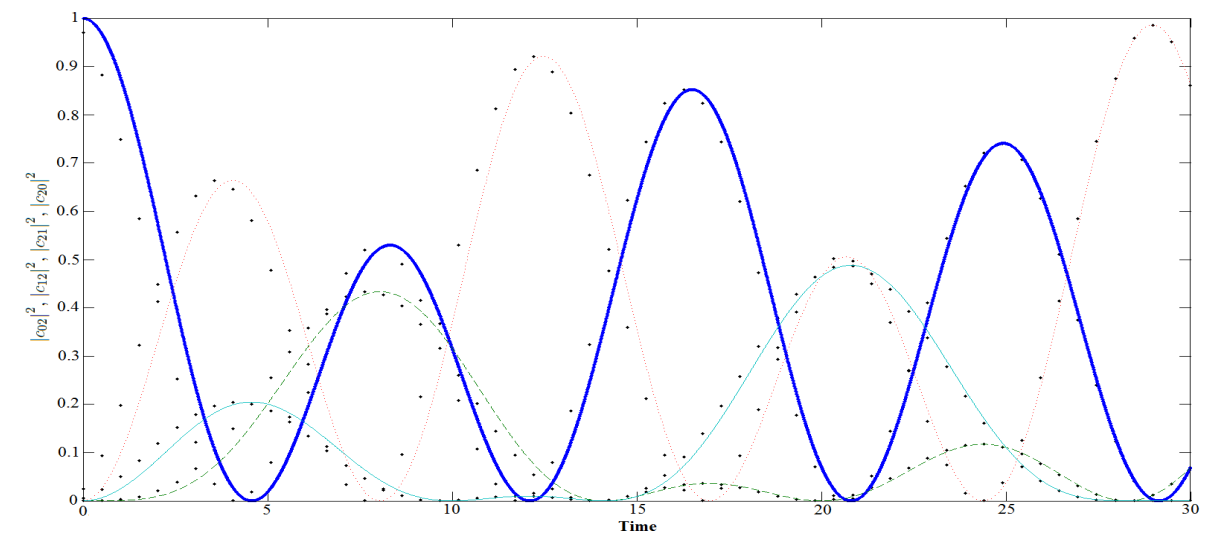

Fig. 3. Probabilities that the system in the $|2\rangle_{a}|0\rangle_{b}$ (bold solid curve), $|2\rangle_{a}|1\rangle_{b}$ (thin solid curve), $|1\rangle_{a}|2\rangle_{b}$ (dashed curve), $|0\rangle_{a}|2\rangle_{b}$ (dot curve) and their numerical results (cross marks). The parameters are the same in the previous figures 


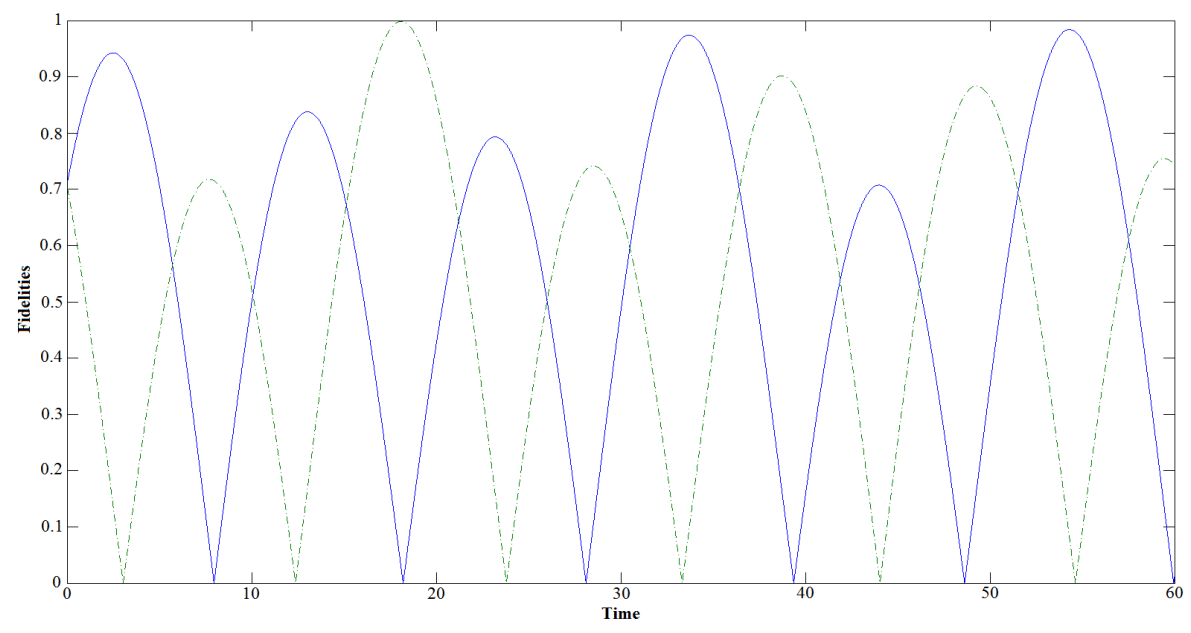

Fig. 4. The fidelities corresponding to the Bell - like $\left|B_{1}\right\rangle$ (solid curve), $\left|B_{2}\right\rangle$ (dot-dashed curve). The parameters are the same in the previous figures

It is easy to see that the maximal entropy of entanglement of the state $|\psi(t)\rangle_{\text {cut }}$ is approximately equal to 1 [ebit] for several moments of time. This result demonstrates the fact that the system behaves as a nonlinear quantum scissor which can be used to generate maximally entangled states. In detail, the main maximum values of entanglement separate from each other with a period $T_{1} \approx 12.5$ ( $1 / \chi$ unit). Besides that, the extra maximum values of entanglement are modulated by an oscillation with period $T_{2} \approx 250$ ( $1 / \chi$ unit). Moreover, time-evolution of entanglement of $|\psi(t)\rangle_{\text {cut }}$ can be described in terms of the qutrit-qutrit system. This manifestation allows us to expect that the Bell-like states may be generated in several times. To check our hypothesis, we calculated the probabilities of observation of the system in the states $|0\rangle_{a}|2\rangle_{b}$, $|2\rangle_{a}|0\rangle_{b},|1\rangle_{a}|2\rangle_{b}$ and $|2\rangle_{a}|1\rangle_{b}$. The results are demonstrated in Fig. 3.

In Fig. 3 we can see in several times that probabilities of states are approximately $\frac{1}{2}$ in pairs, especially for $|0\rangle_{a}|2\rangle_{b}$ and $|2\rangle_{a}|0\rangle_{b}$ states. Consequently, we believe that, the system can generate Bell - like states which have maximal entropy of entanglement equal to 1 [ebit]

$$
\begin{aligned}
& \left|B_{1}\right\rangle=\frac{1}{\sqrt{2}}\left(|2\rangle_{a}|0\rangle_{b}+i|0\rangle_{a}|2\rangle_{b}\right), \\
& \left|B_{2}\right\rangle=\frac{1}{\sqrt{2}}\left(|2\rangle_{a}|0\rangle_{b}-i|0\rangle_{a}|2\rangle_{b}\right) .
\end{aligned}
$$

To show the fact that the creation of Bell - like states $\left|B_{i}\right\rangle$ has been made, we calculate the fidelities $\left|\left\langle\psi(t) \mid B_{i}\right\rangle\right|$ and plot the results in Fig. 4.

Moreover, Fig. 3 shows that, sometimes the probabilities of state pairs $|0\rangle_{a}|2\rangle_{b}$ and $|2\rangle_{a}|1\rangle_{b},|2\rangle_{a}|0\rangle_{b}$ and $|1\rangle_{a}|2\rangle_{b}$ are nearly to $\frac{1}{2}$. Therefore, we can expect that the evolution of the system will generate the states

$$
\begin{aligned}
\left|B_{3}\right\rangle & =\frac{1}{\sqrt{2}}\left(|0\rangle_{a}|2\rangle_{b}+|2\rangle_{a}|1\rangle_{b}\right), \\
\left|B_{4}\right\rangle & =\frac{1}{\sqrt{2}}\left(|0\rangle_{a}|2\rangle_{b}-|2\rangle_{a}|1\rangle_{b}\right), \\
\left|B_{5}\right\rangle & =\frac{1}{\sqrt{2}}\left(|2\rangle_{a}|0\rangle_{b}+|1\rangle_{a}|2\rangle_{b}\right), \\
\left|B_{6}\right\rangle & =\frac{1}{\sqrt{2}}\left(|2\rangle_{a}|0\rangle_{b}-|1\rangle_{a}|2\rangle_{b}\right) .
\end{aligned}
$$

The fidelities corresponding to states $\left|B_{3}\right\rangle,\left|B_{4}\right\rangle,\left|B_{5}\right\rangle$ and $\left|B_{6}\right\rangle$ are plotted in Fig. 5.

It follows from Fig. 4 and Fig. 5 that while the fidelities corresponding states $\left|B_{4}\right\rangle$ and $\left|B_{6}\right\rangle$ are smaller than 0.7 , the fidelities corresponding to states $\left|B_{1}\right\rangle,\left|B_{2}\right\rangle,\left|B_{3}\right\rangle$ and $\left|B_{5}\right\rangle$ have maximal values closed to unity. It means that there exist entangled states in evolution of our system. They are not really Bell-states, but if we increase the coupling length between oscillators, almost all of them have fidelities approximately equal to unity. Consequently, oscillations between states have a bigger frequency and our system behaves as a two qubits system. Moreover, we observe that the results are the same for cases $\beta=\alpha, \beta=-\alpha$ and $\beta=i \alpha$. This means that the time evolution of our system does not depend on the phase deviation between two external fields.

\section{DAMPING CASE}

It is widely known that damping processes can affect physical properties of our system and reduce the maximum values of entanglement. Therefore, in this section we will 


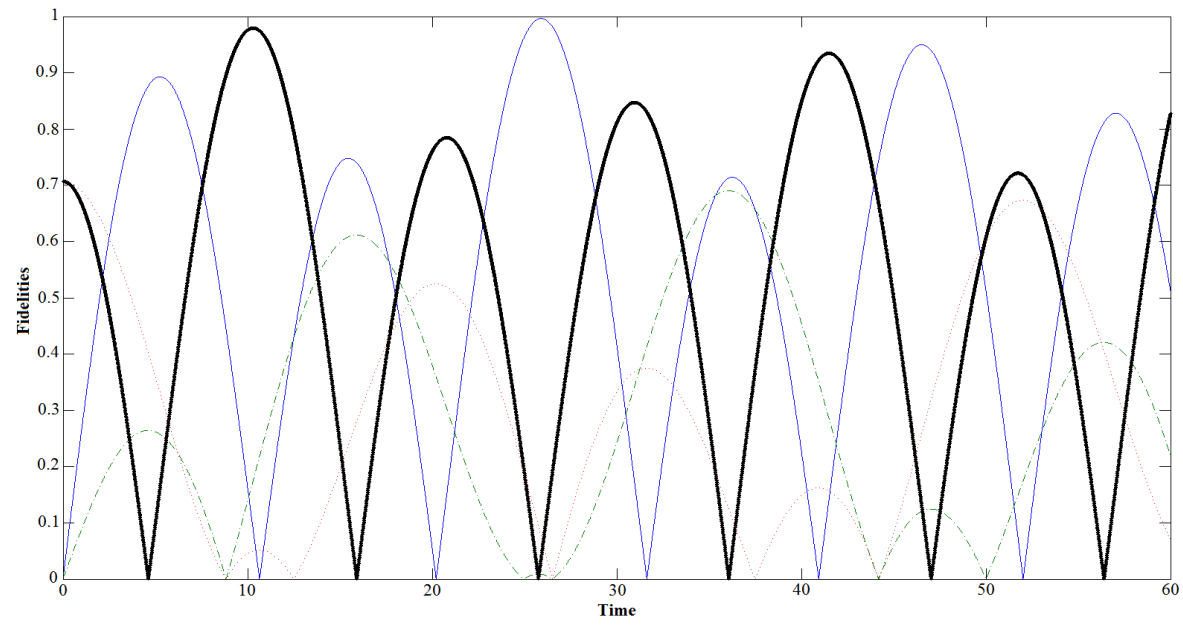

Fig. 5. The fidelities corresponding to the Bell - like $\left|B_{3}\right\rangle$ (thin solid curve), $\left|B_{4}\right\rangle$ (dot-dashed curve), $\left|B_{5}\right\rangle$ (bold solid curve) and $\left|B_{6}\right\rangle$ (dot curve). The parameters are the same in the previous figures

concentrate on the influence of damping processes in the Belllike states creation. Let us assume that the losses of photons in two cavities $a$ and $b$ are characterized by $\kappa_{a}$ and $\kappa_{b}$, respectively. The loss of photons in the cavities corresponds to the annihilation of photons. Therefore, we can consider the collapse operators in the following forms

$$
\begin{gathered}
\hat{C}_{a}=\sqrt{2 \kappa_{a}} \hat{a} \\
\hat{C}_{b}=\sqrt{2 \kappa_{b}} \hat{b} .
\end{gathered}
$$

In this case, the evolution of the system is described by density matrix $\hat{\rho}$ which obeys the master equation

$$
\frac{d \hat{\rho}}{d t}=\hat{\mathcal{L}} \hat{\rho}
$$

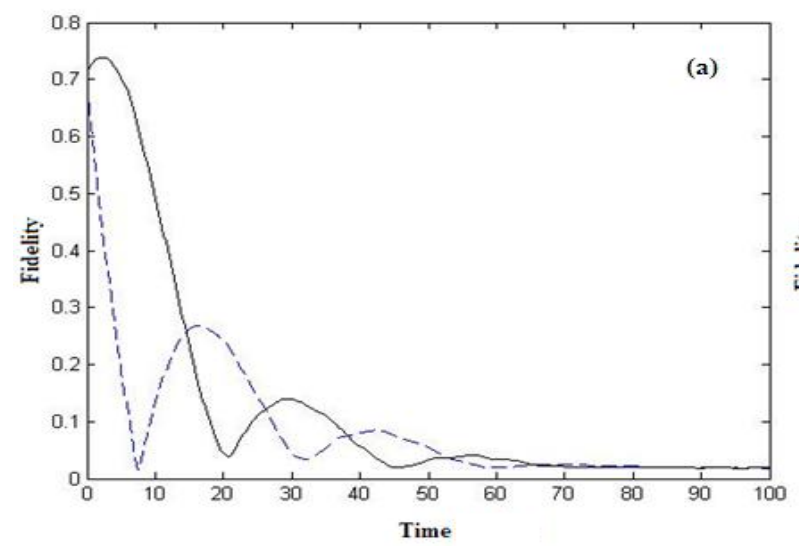

where $\hat{\mathcal{L}}$ is called Liouvillian superoperator. In the Makov approximation, we can write the Liouvillian superoperator $\hat{\mathcal{L}}$ in the form

$$
\hat{\mathcal{L}} \hat{\rho}=-i[\hat{H}, \hat{\rho}]+\hat{\mathcal{L}}_{\text {loss }} \hat{\rho},
$$

where $\hat{\mathcal{L}}_{\text {loss }}$ is the loss term of the Liouvillian superoperator that describes the losses of photons in cavities

$$
\begin{aligned}
\hat{\mathcal{L}}_{\text {loss }} \hat{\rho} & =\hat{C}_{a} \hat{\rho} \hat{C}_{a}^{\dagger}-\frac{1}{2}\left(\hat{C}_{a}^{\dagger} \hat{C}_{a} \hat{\rho}-\hat{\rho} \hat{C}_{a}^{\dagger} \hat{C}_{a}\right)+\hat{C}_{b} \hat{\rho} \hat{C}_{b}^{\dagger} \\
& -\frac{1}{2}\left(\hat{C}_{b}^{\dagger} \hat{C}_{b} \hat{\rho}-\hat{\rho} \hat{C}_{b}^{\dagger} \hat{C}_{b}\right) .
\end{aligned}
$$

In order to find the evolution of the system in this case, we have to know the form of the Liouvillian and solve the

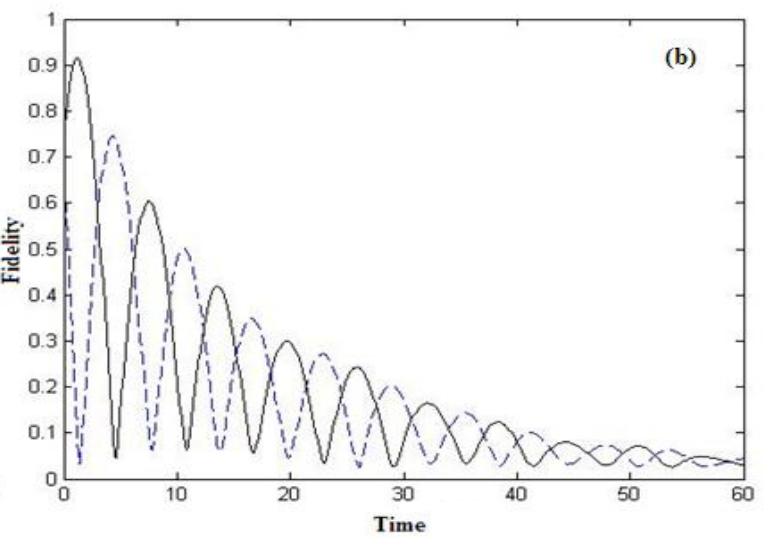

Fig. 6. Fidelities $\mathbf{F}$ corresponding to the Bell-like states $\left|B_{1}\right\rangle$ (solid lines) and $\left|B_{2}\right\rangle$ (dashed lines) for the damping constants $\kappa_{a}=\kappa_{b}=\kappa$, $\kappa=\chi / 75$ (a) and $\kappa=\chi / 500$ (b). Other parameters are remained as in the previous figures. Time unit is scaled in $1 / \chi$ 
master equation numerically. However, the form of master equation (22) suggests that the evolution of our system can be found in a series of potential function

$$
\hat{\rho}(t)=\exp (\hat{\mathcal{L}} t) \hat{\rho}(0),
$$

where $\hat{\rho}(0)$ is the density matrix that describes the state of our system at $t=0$.

Because the generation of Bell-like states achieves the best results with the Bell-like states $\left|B_{1}\right\rangle$ and $\left|B_{2}\right\rangle$, in the further part hereof we will neglect examination for the generation of other Bell-like states and only concentrate on Belllike states $\left|B_{1}\right\rangle$ and $\left|B_{2}\right\rangle$. As it has been mentioned at the beginning of this section, because of the effect of damping processes, we have to describe the evolution of our system by a density matrix instead of a time-dependent wave function. Therefore, in order to find the fidelities corresponding to the Bell-like states, we use the definition of fidelity $F$ in the matrix calculation

$$
F=\operatorname{Tr}\left[\left(\sqrt{\hat{\rho}_{\text {cut }}} \hat{\rho} \sqrt{\hat{\rho}_{\text {cut }}}\right)^{\frac{1}{2}}\right]^{2}
$$

where $\hat{\rho}_{\text {cut }}$ is a density matrix corresponding to the state that is generated by our system and $\hat{\rho}$ is a density matrix corresponding to the compared state. In order to compare our results with the result created by a nonlinear coupler pumped in one mode [10], we will show our results in the same values of the parameters. The fidelities corresponding to the states $\left|B_{1}\right\rangle$ and $\left|B_{2}\right\rangle$ are shown in Fig. 6.

We can see that if the effect of damping processes is small enough (Fig. 6b), the generations of Bell-like states $\left|B_{1}\right\rangle$ and $\left|B_{2}\right\rangle$ are very good. Nevertheless, for the more realistic situation, when the values of damping parameters increase, the fidelities reduce vary rapidly. In order to compare with the results in [10], we change the values of damping parameters to $\kappa_{a}=\kappa_{b}=\kappa=\chi / 75$. The results from Fig. 6a show that when the fidelity corresponding to the state $\left|B_{1}\right\rangle$ increases, the fidelity corresponding to the state $\left|B_{2}\right\rangle$ decreases and vice versa. Moreover, the maximum values of fidelities decrease less suddenly than the case considered in [10]. This is a result of resonance between two fields that pumped to two cavities in addition to the natural resonance of nonlinear interaction between two modes. The results in [19] and [20] have shown a purification scheme that can reduce the effect of damping processes. If we can do that, our system can be seem to be an effective source of maximally entangled states.

\section{CONCLUSIONS}

We have considered a system including nonlinear oscillators that is coupled by nonlinear interaction pumped in two modes. It has been shown that when the coupling constant between modes is much smaller then nonlinearities, the states with large numbers of photons can be neglected and the evolution of the system can be expressed in a set of four states $|0\rangle_{a}|2\rangle_{b},|2\rangle_{a}|0\rangle_{b},|1\rangle_{a}|2\rangle_{b}$ and $|2\rangle_{a}|1\rangle_{b}$. Therefore we can use the nonlinear scissors approach to analyse and obtain the time evolution of system. We have shown that our system can be seem to be a good source of maximally entangled states. The maximally values of entanglement are approximately equal to unity in a larger number of moments of time than the results in [7]. Therefore, our system can generate the Bell-like states with nearly the same results in numerical simulation and analytical calculation. Besides that, our calculations indicate the entanglement and evolution of the system are independent of phase difference between two coherent classical fields which are pumped to modes. The result is different from ones created by nonlinear scissors pumped in two modes with linear interaction between modes [5]. With these specific characteristics, we can believe that our system can not only be applied as a source of entangled states, in particular Bell-like states, but also as an element of more complex systems used in quantum computation.

\section{Acknowledgments}

We are grateful to prof. Wieslaw Leoński for his valuable discussions.

\section{References}

[1] M. A. Nielsen and I. L. Chuang, Quantum Computation and Quantum Information, Cambridge University Press, 7190 (2000).

[2] M. Le Bellac, A Short Introdution To Quantum Information and Quantum Computation, Cambridge University Press, 49-54 (2006).

[3] S. Babichev, J. Ries and A. Lvovsky, Quantum scissors: Teleportation of single-mode optical state by means of nonlocal single photon, Europhys. Lett. 64, 1-7. (2003).

[4] E. Bimbard, N. Jain, A. MacRae and A.I. Lvovsky, Quantumoptical state engineering up to the two-photon levels. Nature Photonic, 10, 243-247 (2010).

[5] A. Miranowicz and W. Leonski, Two-mode optical state truncation and generation of maximally entangled states in pumped nonlinear couplers, Phys. B: At. Mol. Opt. Phys. 39, 1683-1700 (2006).

[6] W. Leonski, Kicked nonlinear quantum scissors and entanglement generation, Phys. Rev. A 55, 4250-4265 (1997).

[7] A. Kowalewska-Kudlaszyk and W. Leonski, Finitedimensional states and entanglement generation for a nonlinear coupler, Phy. Rev. A 73, $042318-042334$ (2006).

[8] N. T. Dung, W. Leonski, V. Cao Long, Computer Simulation of Two-mode Nonlinear Scissors, Comp. Meth. Sci. Technol. Vol 19 (3), 175-181 (2013).

[9] A. Kowalewska-Kudlaszyk, W. Leonski, V. Cao Long, N.T Dung, Kicked nonlinear quantum scissors and entanglement generation, Phys.Scr. 160, 014023-014032 (2014).

[10] A. Kowalewka - Kudlaszyk and W. Leonski, Exciting field phase effect on the entanglement death and birth phenomena for nonlinearcoupler system, Journal of Computational Methods in Sciences and Engineering, vol.10, no. 3-6, 425-431 (2010) 
[11] A. Kowalewska-Kudlaszyk and W. Leonski, Sudden death and birth of entanglement effects for Kerr-nonlinear coupler, J. Opt. Soc. Am. B Vol 26. 7, 1289-1294 (2009)

[12] A. Kowalewska-Kudlaszyk and W. Leonski, Sudden death of entanglement and its rebirth in a system of two nonlinear oscillators, Phys. Scr. T140, 014051-10456 (2010).

[13] D. T. Pegg, L.S. Phillips and S. M. Barnett, Quantum scissors: teleportation of single-mode optical states by means of a nonlocal single photon, Phys. Rev. Lett. 81, 1604-1608 (1998).

[14] W. Leonski, S. Dyrting and R. Tanas, Fock states generation in a kicked cavity with a nonlinear medium, J. Mod. Opt. 44, 2105-2123 (1997).

[15] W. Leonski and A. Miranowicz, Kerr nonlinear coupler and entanglement, J. Opt. B: Quantum Semiclassical Opt. 6, S37S42 (2004).

[16] A. Kowalewka - Kudlaszyk and W. Leonski, A Quantum scissors finite-dimensional states engineering, , Progress in Optics, vol 56, 131-185 (2011).

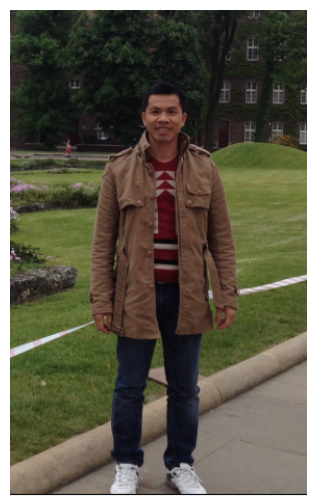

V. Le Duc was born in Thanh Hoa province, Vietnam. He graduated in physics in 2005 at Hue University and finished his MSc study in 2015 at the Hong Duc University under supervision of prof. Cao Long Van. He is interested in quantum computation, quantum information and nonlinear optics.
[17] W. K. Wootters, Entanglement of formation of an arbitrary state of two qubits, Phys. Rev. Lett, 80, 2245 - 2251 (1998).

[18] G. Jaeger, Entanglement information and interpretation of quantum mechanics, Springer Press, 12-24 (2009)

[19] A. Kent, Entangled Mixed States and Local Purification Phys. Rev. Lett. 81, 2839-2844 (1998).

[20] R. Horodecki, M. Horodecki and P. Horodecki, General teleportation channel, singlet fraction, and quasidistillation, Phys. Rev. A. 60, 4144-4150 (1999).

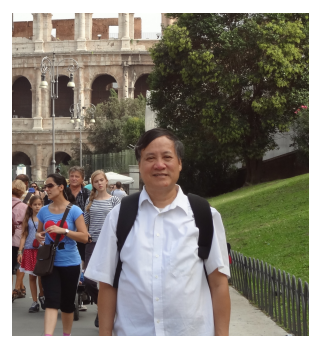

V. Cao Long was born in 1952 in Hanoi, Vietnam. He graduated from Warsaw University with an MSc degree in theoretical physics. In 1979 he defended his PhD dissertation at this University. In 1987 he habilitated in the Center of Theoretical Physics, Polish Academy of Sciences in Warsaw with a thesis entitled "Noises in Quantum Optics". He is presently associate professor of the University of Zielona Góra. His research interests concern several topics in quantum and nonlinear optics. 